\title{
Iron(III)-Catalyzed Enantioselective Synthesis of Allenes
}

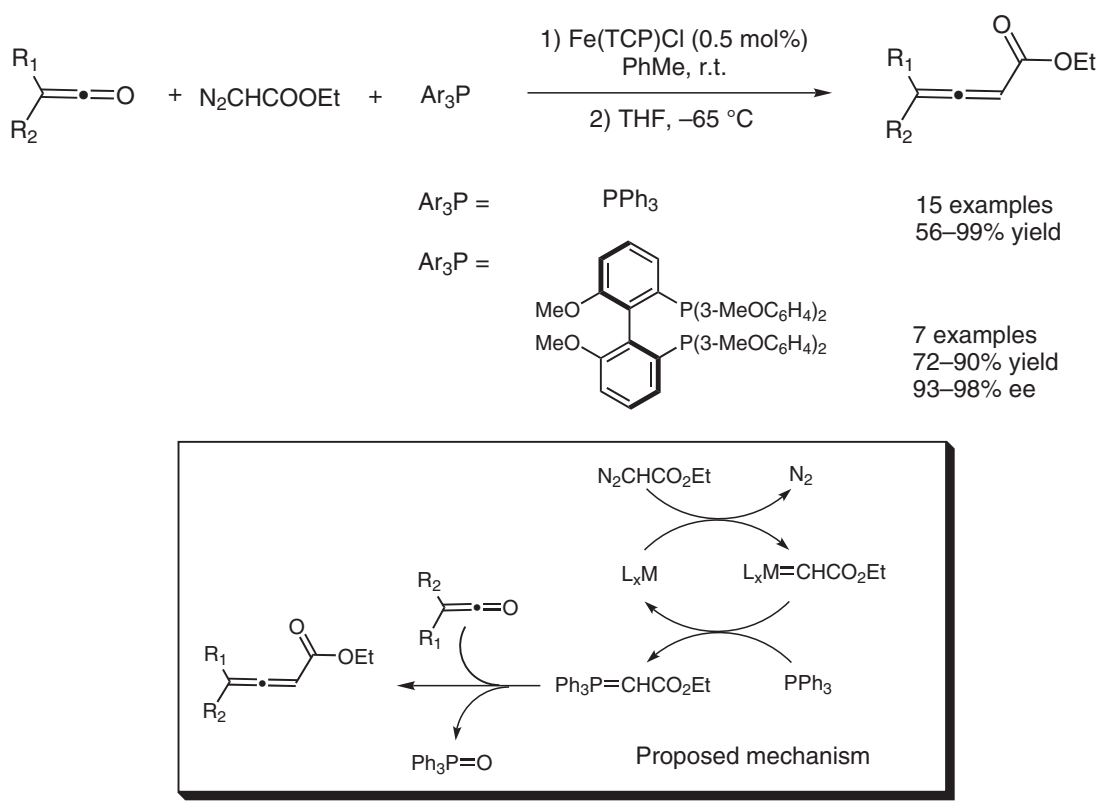

Significance: Olefination of ketenes with ethyl diazoacetate using an Fe(III) catalyst and triphenylphosphine provides an efficient synthesis of 4-monosubstituted and 4,4-disubstituted allenic esters. Only 0.5 mol\% of $\mathrm{Fe}(\mathrm{TCP}) \mathrm{Cl}$ is required and the reaction conditions are mild. The phosphine ligand can also be recovered and reused. The scope of the reaction is broad and encompasses a variety of substituents on the ketene, including $\mathrm{H}, \mathrm{Br}$, alkyl and aryl groups. A one-pot strategy has also been developed for unstable ketenes by synthesizing the ketene in situ. An asymmetric version is possible by using a chiral phosphine ligand, and affords chiral allenes in good yields and excellent enantioselectivities.
Comment: Although many procedures for the synthesis of allenes have been reported, it remains challenging to find procedures that are mild and practical, especially for chiral allenes. The present report is thus important for making optically active allenes under neutral conditions. The high ee reported for the use of chiral phosphines supports the mechanistic hypothesis of Woo and co-workers (G.A. Mirafzal, G. Cheng, L.K. Woo J. Am. Chem. Soc. 2002, 124, 176-177), who suggested that the free ylide is formed. Catalytic transfer from Fe(III) to phosphine would yield the free ylide. A study has also been performed in the present report to show that a monoylide is the likely intermediate. 